\title{
Faraday tomography for magnetic fields in our Galaxy and nearby Universe
}

\author{
JinLin Han* \\ National Astronomical Observatories, Chinese Academy of Sciences, \\ Jia-20, DaTun Road, Chaoyang District, Beijing 100012, China \\ E-mail: hjl e bao.ac.cn
}

To understand the magnetic fields in the universe, we have to measure them and get to know their properties. The magnetic fields of galactic scales are crucial bridge to connect the knowledge on the stellar scale and the cosmological scale. The most effective method to reveal the large-scale extremely weak magnetic fields on galactic or a larger scale is through observing the Faraday rotation of the polarized emission of the background radio sources. Note that the Faraday rotation is integration of the magnetic field $B$ together with the thermal electron density $n_{e}$. In our own Milky Way Galaxy, pulsars are the best probes, because we can observe not only the rotation measure (RM), but also the pulse delay at different frequencies, i.e. the dispersion measure (DM). From these two observables, the magnetic field in the interstellar medium can be directly measured. In the Galactic disk, we obtained the large-scale magnetic field structure from pulsar RMs and DMs, and also we determined the spatial magnetic field energy spectrum which theoretic studies need. The Galacto-radial dependence of the field strength has also been derived from pulsar data. From the sky distribution of RMs of extragalactic radio sources, we identified the magnetic field structure in the Galactic halo. To explore the magnetic fields of cosmological scale, the RMs in the Galactic pole regions can be used, because the foreground RM contribution from our Galaxy is minimized and can be eliminated easily. There is evidence that the RMs become more deviate towards higher redshift, i.e. there are intergalactic magnetic fields but it is hard to disentangle it from the combination with unknown electron distribution in the intergalactic space.

From Planets to Dark Energy: the Modern Radio Universe

October 1-5 2007

The University of Manchester, $U K$

\footnotetext{
* Speaker.
} 


\section{Introduction}

Our own Galaxy, the Milky way, is so bright in sky in optical and radio bands, which we all know about it. However, the polarized radio sky [20, 22] and the Faraday's sky or the Rotation Measure (RM) Sky are not so familiar for many astronomers except for some radio astronomers. Our Galaxy is dominant in the sky, and the RM sky has a strikingly antisymmetric distribution [9]. The foreground contribution from our Galaxy, or "pollution", must be eliminated when we like measure any polarization or Faraday effect on the cosmological scale. However, the foreground contribution has not been measured exactly, especially the RM sky.

Faraday rotation of polarized emission from a radio source can be used to reveal the intervening magnetic fi elds between the source $(*)$ and us $(\oplus)$. The rotation of polarization plane caused by the magnetized medium is

$$
\psi=810 \int_{\oplus}^{*} \lambda^{2}(l) n_{e}(l) \mathbf{B}(l) \cdot d \mathbf{l},
$$

here, $\psi$ is total rotation angle (in rad), $\lambda$ is wavelength (in $\mathrm{m}$ ), $n_{e}(l)$ is intervening electron density (in $\mathrm{cm}^{-3}$ ), $\mathbf{B}$ is vector magnetic fi eld (in $\mu \mathrm{G}$ ), and $d \mathbf{l}$ is the unit vector of the line of sight (in $\mathrm{kpc}$ ) pointing towards us. Note that electron density and magnetic fi eld vary along the line of sight. To reveal the intervening magnetic fields, it is necessary to know the distribution of electron density along the line of sight. For a cosmological radio source at a given location in the universe, e.g. at redshift $z$, the wavelength $\lambda(z)$ is also specifi cally related to observed wavelength by $\lambda_{\text {obs }}=$ $(1+z) \lambda(z)$. So, the rotation measure $\left(\mathrm{RM}\right.$, in $\left.\mathrm{rad} \mathrm{m}^{-2}\right)$ of a radio source at a redshift, $z_{s}$, should be defi ned as

$$
R M_{\mathrm{obs}}=\frac{\psi_{1}-\psi_{2}}{\lambda_{\mathrm{obs} 1}^{2}-\lambda_{\mathrm{obs} 2}^{2}}=810 \int_{0}^{z_{s}}(1+z)^{-2} n_{e}(z) \mathbf{B}(z) \cdot d \mathbf{l} .
$$

In different cosmological models, the $d l$ and $d z$ are related by

$$
\frac{d l}{d z}=\frac{c}{H_{0}}(1+z)^{-1}\left[\Omega_{\mathrm{m}}(1+z)^{3}+\left(1-\Omega_{\mathrm{m}}-\Omega_{\Lambda}\right)(1+z)^{2}+\Omega_{\Lambda}\right]^{-1 / 2} .
$$

Here, $H_{0}=100 h \mathrm{~km} \mathrm{~s}^{-1} \mathrm{Mpc}^{-1}$ is the Hubble constant, $h$ is the dimensionless factor, $c$ is the speed of light, $\Omega_{\mathrm{m}}$ is the dimensionless ordinary matter density, and $\Omega_{\Lambda}$ is the vacuum energy density. Most recent measurements show that $h=0.72 \pm 0.05, \Omega_{\mathrm{m}} \sim 0.3$ and $\Omega_{\Lambda} \sim 0.7$ [ [, 23 ].

Observed Faraday rotation consists of three contributions,

$$
R M_{\mathrm{obs}}=R M_{\mathrm{in}}+R M_{\mathrm{ig}}+R M_{\mathrm{fg}}
$$

namely, the intrinsic rotation measure local to a source, $R M_{\mathrm{in}}$, the rotation measure from the intergalactic medium, $R M_{\mathrm{ig}}$, and the foreground $\mathrm{RM}$ from our Galaxy, $R M_{\mathrm{fg}}$. The foreground $\mathrm{RM}$ from our Galaxy are common contribution to RMs of radio sources located in a small sky region. To reveal the properties of intergalactic magnetic fi elds, the effect of intrinsic and foreground rotation measures should be eliminated from the observed values of $R M_{\mathrm{obs}}$.

Pulsars in our own Galaxy emit polarized radio emission, and their RMs can be used to measure the interstellar magnetic fi elds $[1 \phi]$. Observed Faraday rotation of pulsars does not have any intergalactic contribution or intrinsic contribution, nor do we have to consider the cosmological 
effect on the wavelength. Therefore, the $R M$ (in radians $\mathrm{m}^{-2}$ ) of a pulsar at distance $D$ (in $\mathrm{kpc}$ ) can be simply given by $\mathrm{RM}=810 \int_{0}^{D} n_{e} \mathbf{B} \cdot d \mathbf{l}$. Positive $R M$ s correspond to the average fi elds directed toward us. In addition, the electron density between a pulsar and us can be measured by the pulse delay between the high and low radio frequencies (even at the two ends of a receiving radio wave band). This is the dispersion measure (DM) of a pulsar, $\mathrm{DM}=\int_{0}^{D} n_{e} d l$. From the two observables, $D M$ and $R M$, we obtain a direct estimate of the fi eld strength weighted by the local free electron density,

$$
\left\langle B_{\|}\right\rangle=\frac{\int_{0}^{D} n_{e} \mathbf{B} \cdot d \mathbf{l}}{\int_{0}^{D} n_{e} d l}=1.232 \frac{\mathrm{RM}}{\mathrm{DM}} .
$$

where RM and DM are in their conventional units of $\operatorname{rad~m}^{-2}$ and $\mathrm{cm}^{-3} \mathrm{pc}$ and $B_{\|}$is in $\mu \mathrm{G}$.

\section{RM tomography for magnetic fields in the Galactic disk}

Pulsars are best probes for the Galactic magnetic fi elds. Magnetic fi elds in a large-part of the Galactic disk have been delineated by Faraday rotation data of pulsars, which gives a very direct estimate of the line-of-sight component of the magnetic fi eld through normalization by the dispersion measure (DM). They typically have substantial linear polarization, making the Faraday rotation relatively easy to measure; they are distributed in the Galaxy with approximately known distances, allowing the three-dimensional properties of the magnetic fi eld to be investigated; and they apparently have no intrinsic rotation measure. RMs of extragalactic sources can be also used to reveal the Galactic magnetic fi elds. The RM average over a number of sources in a given sky region should be the common RM contribution from the Galactic disk, i.e. the integral of $n_{e} \mathbf{B}$ from the boundary of the Galactic disk toward us along the line of sight. Interpretation of extragalactic $\mathrm{RM}$ data [3] are then rested on the model of $n_{e}$ and $\mathbf{B}$ in the interstellar space. However, these extragalactic RMs are unique probes for magnetized interstellar medium beyond the known pulsars up to the disk boundary.

\subsection{Magnetic field structure in the Galactic disk}

Previous analysis of pulsar RM data often used the model-fi tting method [13,19], i.e., to model magnetic fi eld structures in the all paths from pulsars to us (observer) and fit them together with the electron density model to observed RM data. Significant improvement can be obtained now when RM and DM data are available for many pulsars in a given region with similar lines of sight. Measuring the gradient of RM with distance or DM is the most powerful method of determining both the direction and magnitude of the large-scale fi eld in that particular region of the Galaxy [17. 11]. Field strengths in the region can be directly measured (instead of modeled) from the slope of trends in plots of RM versus DM. Based on Equation 1.5, we get

$$
\left\langle B_{\|}\right\rangle_{d 1-d 0}=1.232 \frac{\Delta \mathrm{RM}}{\Delta \mathrm{DM}}
$$

where $\left\langle B_{\|}\right\rangle_{d 1-d 0}$ is the mean line-of-sight fi eld component in $\mu \mathrm{G}$ for the region between distances $d 0$ and $d 1, \Delta \mathrm{RM}=\mathrm{RM}_{d 1}-\mathrm{RM}_{d 0}$ and $\Delta \mathrm{DM}=\mathrm{DM}_{d 1}-\mathrm{DM}_{d 0}$.

Up to now, RMs of 550 pulsars have been observed [5, 12, 26, 11]. Most of the new measurements lie in the fourth and fi rst Galactic quadrants and are relatively distant, which enable us 


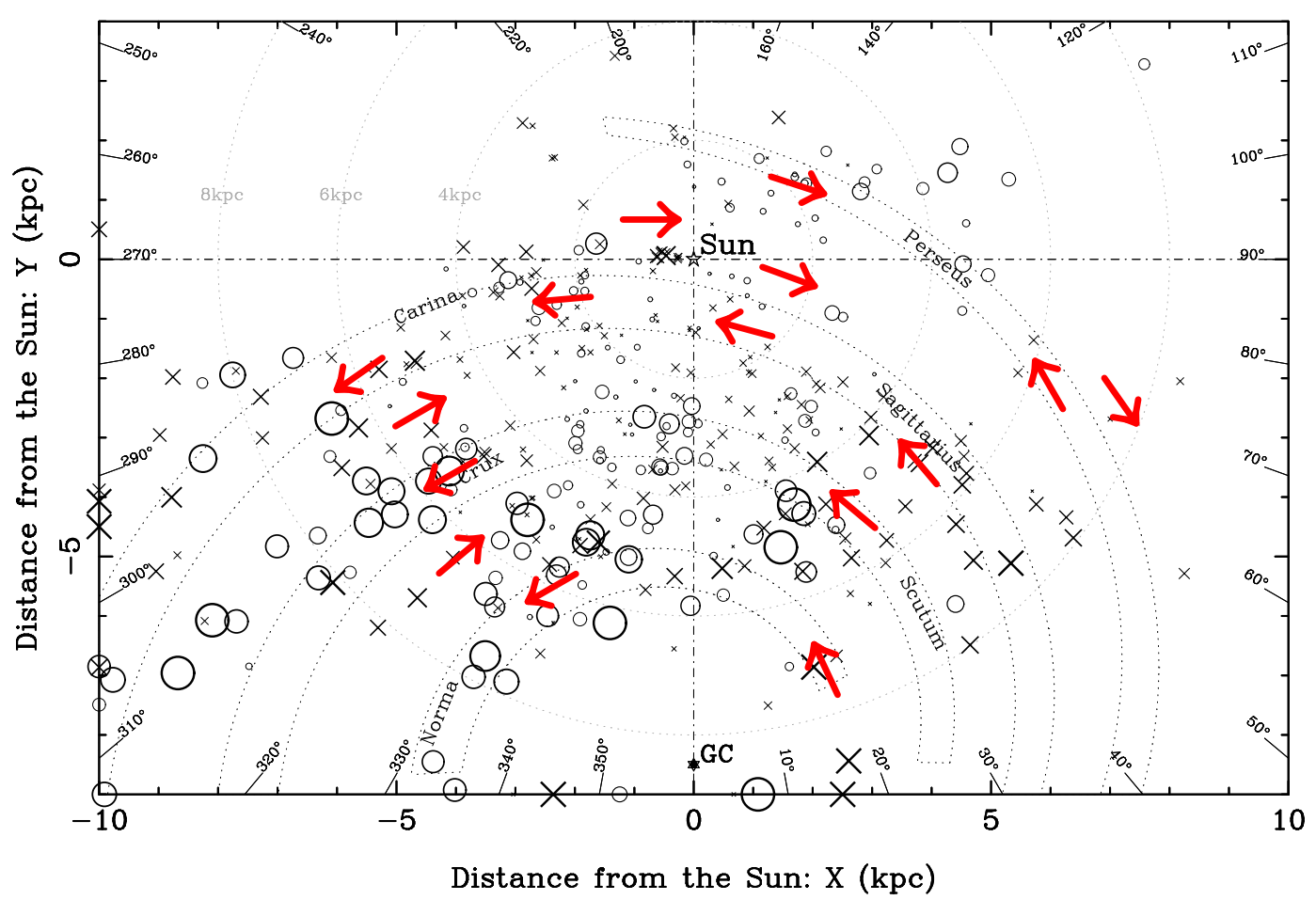

Figure 1: The RM distribution of 374 pulsars with $|b|<8^{\circ}$, projected onto the Galactic Plane. The linear sizes of the symbols are proportional to the square root of the RM values. The crosses represent positive RMs, and the open circles represent negative RMs. The approximate locations of four spiral arms are indicated. The large-scale structure of magnetic fields derived from pulsar RMs [11] are indicated by thick arrows.

to investigate the structure of the Galactic magnetic fi eld over a much larger region than was previously possible. We detected counterclockwise magnetic fi elds in the most inner arm, the Norma arm [10]. A more complete analysis for the fi elds near the tangential regions of the most probable spiral of our Galaxy [11] gives such a picture for the coherent large-scale fi elds aligned with the spiral-arm structure in the Galactic disk, as shown in Fig.1: magnetic fi elds in all inner spiral arms are counterclockwise when viewed from the North Galactic pole. On the other hand, at least in the local region and in the inner Galaxy in the fourth quadrant, there is good evidence that the fi elds in interarm regions are similarly coherent, but clockwise in orientation. There are at least two or three reversals in the inner Galaxy, probably occurring near the boundary of the spiral arms. The magnetic fi eld in the Perseus arm can not be determined well. The negative RMs for distant pulsars and extragalactic radio sources [2] (see Fig. 1) in fact suggest the interarm fi elds both between the Sagittarius and Perseus arms and beyond the Perseus arm are predominantly clockwise.

The average RM variation along the Galactic longitude of extragalactic radio sources [3], especially these of the fourth Galactic quadrant, are very consistent with the magnetic fi eld directions derived from the tangential regions of the arms (see Fig. 2). This implies that the dominant contribution to RMs of extragalactic radio sources behind the Galactic disk comes from the interstellar medium mainly in tangential regions. 

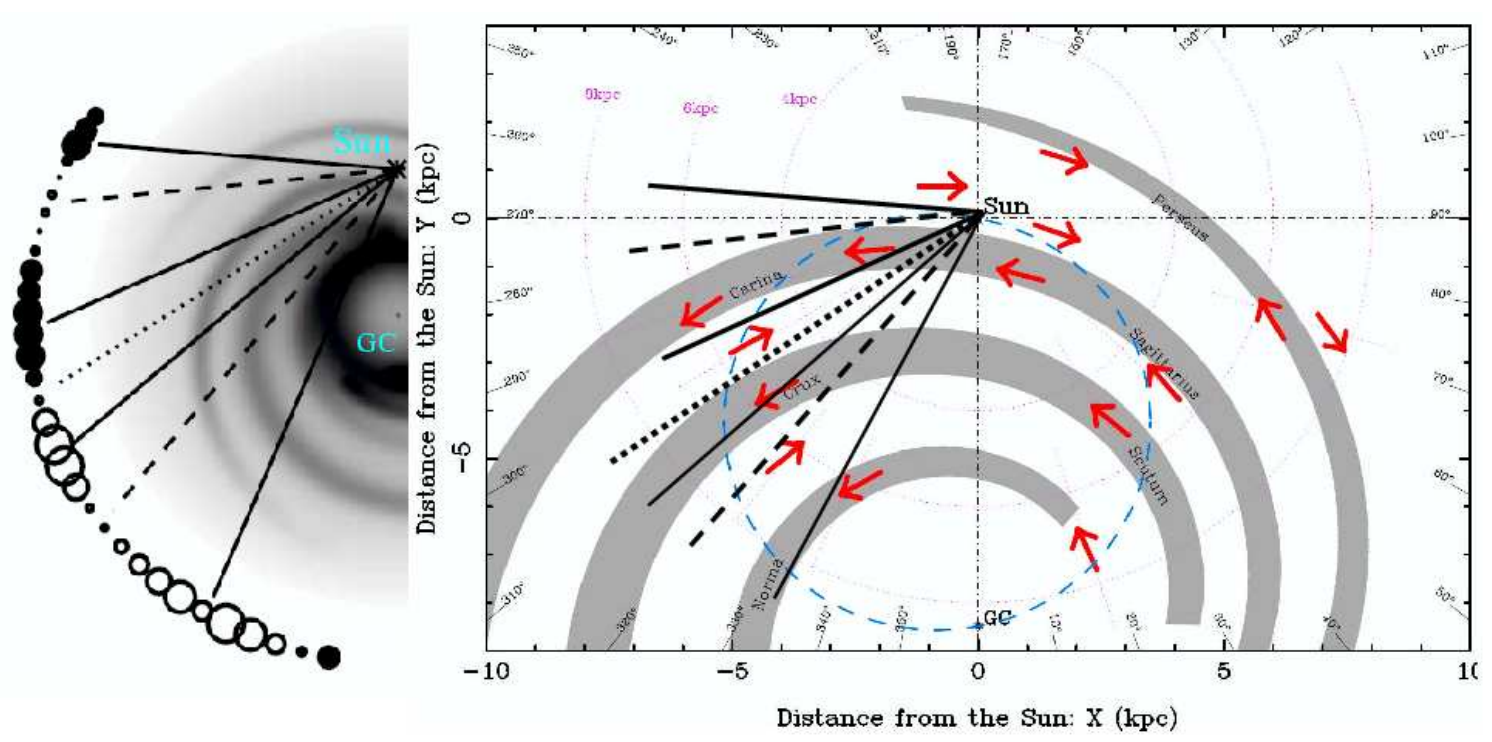

Figure 2: The general tendency of RM variations of extragalactic radio sources along the Galactic longitude, peaks and valleys [3], is very consistent with the large-scale structure of magnetic fields in the tangential regions derived from pulsar RMs [11].

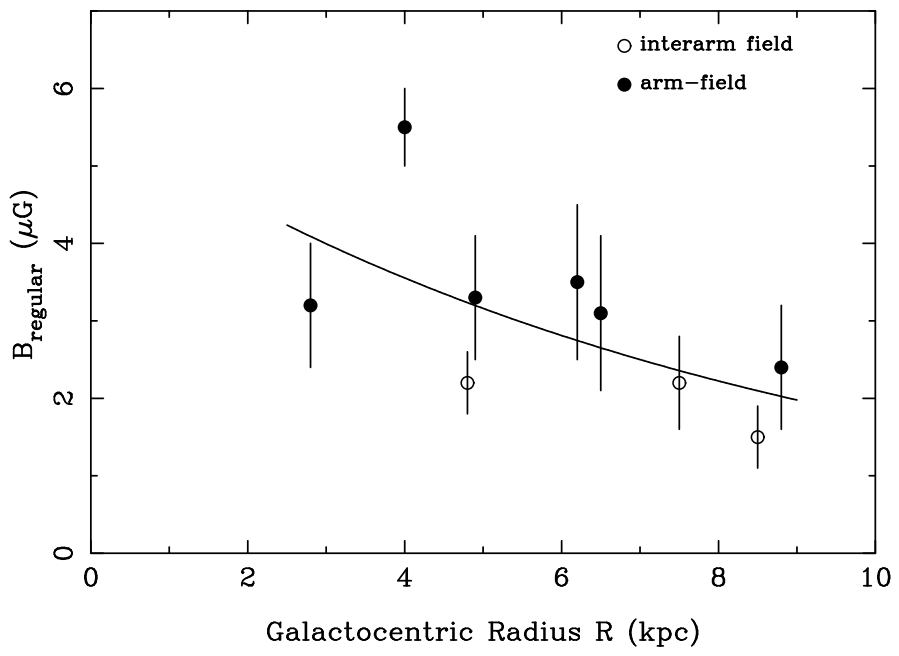

Figure 3: Variation of the large-scale regular field strength with the Galactocentric radius derived from pulsar RM and DM data near the tangential regions [11]. Note that the "error-bars" are not caused by the uncertainty of the pulsar RM or DM data, but reflect the random magnetic fields in the regions.

\subsection{Dependence of the field strength on the Galactocentric radius}

Stronger regular magnetic fi elds in the Galactic disk towards the Galactic Center have been suggested previously [14, 24,. Measurements of the regular fi eld strength in the Solar vicinity give values of $1.5 \pm 0.4 \mu \mathrm{G}$ [21, 13, 15], but near the Norma arm it is $4.4 \pm 0.9 \mu \mathrm{G}$ [10].

With signifi cant more pulsar RM data now available, Han et al. were able to measure the regular fi eld strength near the tangential points in the 1st and 4th Galactic quadrants [11], and then plot the dependence of regular fi eld strength on the Galactoradii (see Fig. 3). Although uncertainties are large, there are clear tendencies for fi elds to be stronger at smaller Galactocentric radii and 
weaker in interarm regions. To parameterize the radial variation, an exponential function was used as following, which not only gives the smallest $\chi^{2}$ value but also avoids the singularity at $R=0$ (for $1 / R$ ) and unphysical values at large $\mathrm{R}$ (for the linear gradient). That is,

$$
B_{\text {reg }}(R)=B_{0} \exp \left[\frac{-\left(R-R_{\odot}\right)}{R_{\mathrm{B}}}\right],
$$

with the strength of the large-scale or regular fi eld at the Sun, $B_{0}=2.1 \pm 0.3 \mu \mathrm{G}$ and the scale radius $R_{\mathrm{B}}=8.5 \pm 4.7 \mathrm{kpc}$.

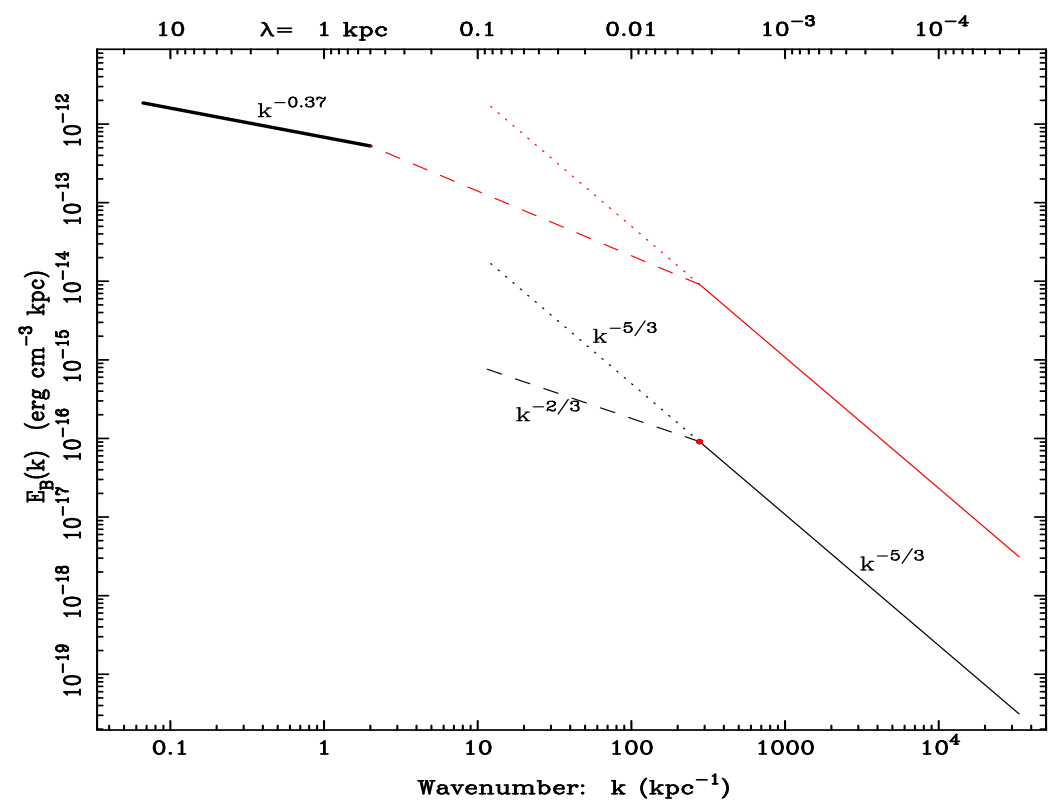

Figure 4: Composite magnetic-energy spectrum in our Galaxy. The large-scale spectrum was derived from pulsar RM data [ 仏. The thin solid and dashed/dotted lines at smaller scales are the Kolmogorov and 2Dturbulence spectra given by Minter \& Spangler [18], and the upper one is from new measurements of Minter (2004, private email).

\subsection{Field strength on different scales}

Interstellar magnetic fi elds exist over a broad range of spatial scales, from the large Galactic scales to the very small dissipative scales, but with different fi eld strength. Knowledge of the complete magnetic energy spectrum can offer a solid observational test for dynamo and other theories for the origin of Galactic magnetic fi elds.

Estimation of the large-scale fi eld strength [13,11] and a turbulent fi eld strength at a scale of tens of pc [21, 19] is only the first step. The spatial power spectrum of electron density fluctuations from small scales up to a few pc [1] could be approximated by a single power law with a 3D spectral index -3.7 , very close to the Kolmogorov spectrum, which gives us a hint that the magnetic energy on the small scales to a few pc should have the Kolmogorov spectrum as well. This was confi rmed by Minter \& Spangler who found [18] that structure functions of RM and emission measure were consistent with a 3D-turbulence Kolmogorov spectra of magnetic fi elds up to $4 \mathrm{pc}$, but with a 2D turbulence between $4 \mathrm{pc}$ and $80 \mathrm{pc}$ (see Fig. 4). 
Pulsar RMs are the integration of fi eld strength together with electron density over the path from a pulsar to us. Therefore, RM data of pulsars with different distances should reflect the fluctuations on different scales. Han et al. [7] used not only pulsars but also pulsar pairs with similar lines of sight to calculate the magnetic energy associated with each pulsar or pulsar pair. Using these pulsars and pulsar pairs, the averaged magnetic energy and the "differential" magnetic energy can be obtained at various scales, and then the spatial energy spectrum of the Galactic magnetic fi eld in scales between $0.5<\lambda<15 \mathrm{kpc}$ can be derived [7]. The result is a 1D power-law, $E_{B}(k) \sim$ $k^{-0.37 \pm 0.10}$, with $k=1 / \lambda$ (see Fig. 4). The rms fi eld strength is approximately $6 \mu \mathrm{G}$ over the relevant scales and the spectrum is much flatter than the Kolmogorov spectrum for the interstellar electron density and magnetic energy at scales less than a few pc. This study complements the derivation of the magnetic energy spectrum over the scale range $0.03-100 \mathrm{pc}$ by [18], showing that the magnetic spectrum becomes flatter at larger scales.

\section{RM tomography for magnetic fields in the Galactic halo}

The magnetic fi eld structure in halos of other galaxies is diffi cult to observe. Our Galaxy is a unique case for detailed studies, since polarized radio sources all over the sky can be used as probes for the magnetic fi elds in the Galactic halo.

As we mentioned before, the foreground RM from our Galaxy are common contribution to RMs of radio sources. That is to say, an "averaging process", which eliminates the random intrinsic RMs and discards the anonymous RMs, should be used to reveal the Galactic RM contribution. We removed any source if its RM value deviates from the average of their neighbours by 3 sigma, i.e. fi ltering out the outliers of RM values that is probably signifi cantly from intrinsic RM, then from such a "cleaned" RM distribution in the sky, Han et al. identifi ed the striking antisymmetry in the inner Galaxy respect to the Galactic coordinates [9, 12]. This RM sky can result from the azimuth magnetic fi elds in the Galactic halo with reversed fi eld directions below and above the Galactic plane (see Fig.2). Such a fi eld can be naturally produced by an A0 mode of dynamo (see reference [27] for a review), and it is necessary to include this into any reasonable model for interstellar medium [25]. The observed fi laments near the Galactic center should result from the dipole fi eld in this scenario. The local vertical fi eld component of $\sim 0.2 \mu \mathrm{G}[13,113]$ may be related to the dipole fi eld in the solar vicinity.

I have shown [6] that the RM amplitudes of extragalactic radio sources in the mid-latitudes of the inner Galaxy are systematically larger than those of pulsars, indicating that the antisymmetric magnetic fi elds are not local but are extended towards the Galactic center, far beyond the pulsars. We are observing more RMs of extragalactic radio sources and modeling the RM sky with a various magnetic fi eld structure in the Galactic halo. For example, the azimuthal halo fi elds with reversed directions above and below the Galactic plane could simply result from a shearing of the dipole fi elds by differentially rotating layers of the ISM.

\section{RM tomography for magnetic fields in the nearby universe}

To probe the magnetic fi elds on cosmological scales, we have to look at the variation of RMs of radio sources with the redshift of the sources after the foreground Galactic RM contribution is 


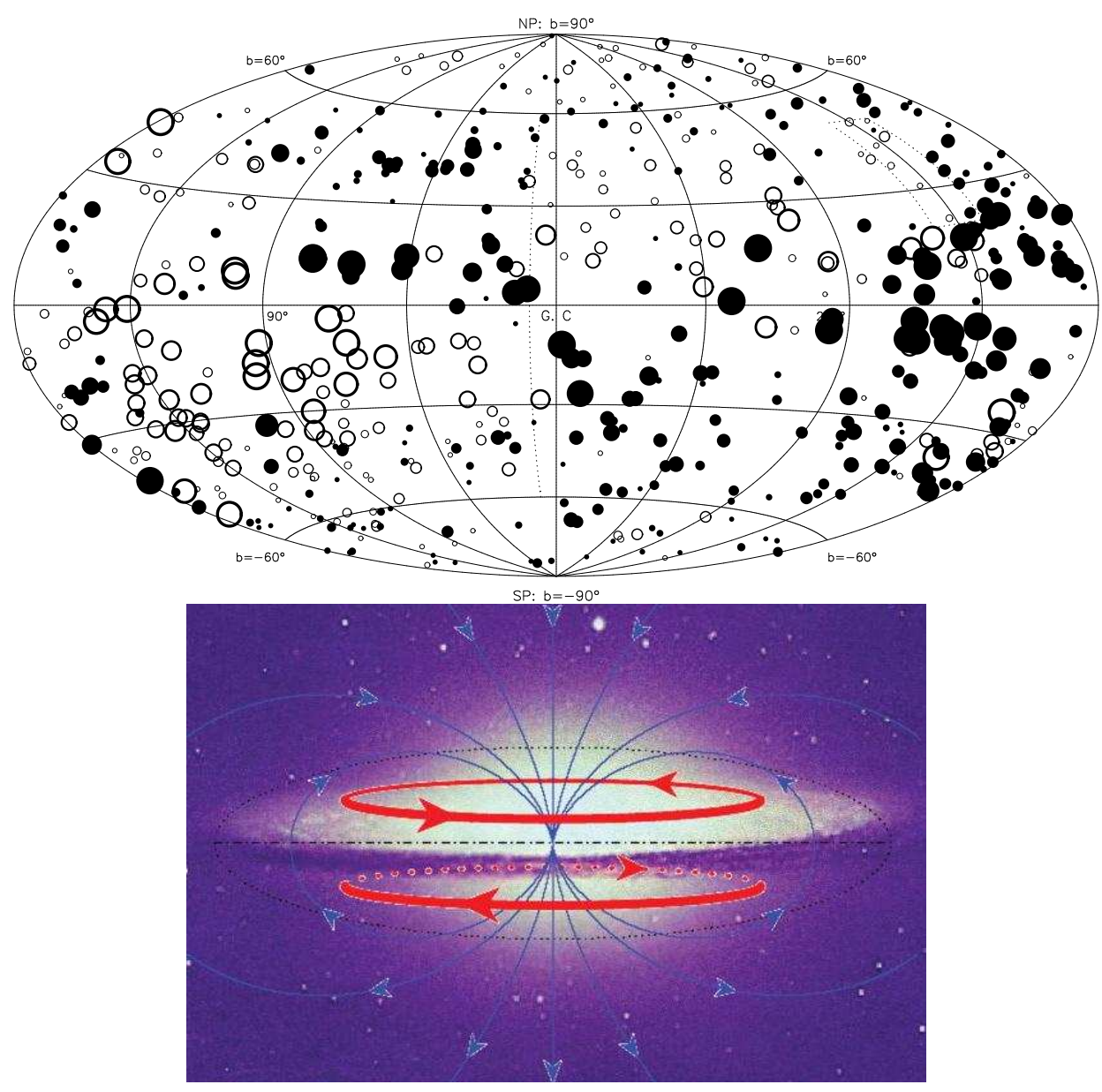

Figure 5: The antisymmetric rotation measure sky, derived from RMs of extragalactic radio sources after filtering out the outliers of anomalous RM values, should correspond to such a magnetic field structure in the Galactic halo as illustrated [9, 12].

eliminated. Three conditions have to be satisfi ed: 1). We have to measure the foreground RM sky to a certain level of accuracy. Looking at the RM sky in Fig.5, one can immediately see that the RMs near the two Galactic poles are on average very small. Whilst in other regions, the Galactic RMs are more diffi cult to assess accurately. A more extensive RM sky survey is required for this purpose. 2). To reveal the intergalactic RMs of a few $\operatorname{rad} m-2$, the measurements of each RM should be at least accurate to this level. With current technicques this is now acheivable using the wide-band spectral-polarimeters available at many radio telescopes. 3). The redshift of measured objects must also be known. This is now becoming possible for large numbers of sources by virtue of new large-scale optical spectrum survey, such as SDSS.

We have observed 110 objects with known redshift in the pole regions [8]. Together with previously published data, we found from RM data of two poles, see Fig. 6, that: 1). RM data clearly tend to have opposite signs which indicate a small but signifi cant local vertical Galactic magnetic fi elds of $0.2 \mu \mathrm{G} ; 2$ ) the deviations get larger at higher redshifts, which implies clearly that there is some kind of random RM contribution from intergalactic medium.

To understand the intergalactic magnetic fi elds, we still have three barriers to overcome. In 


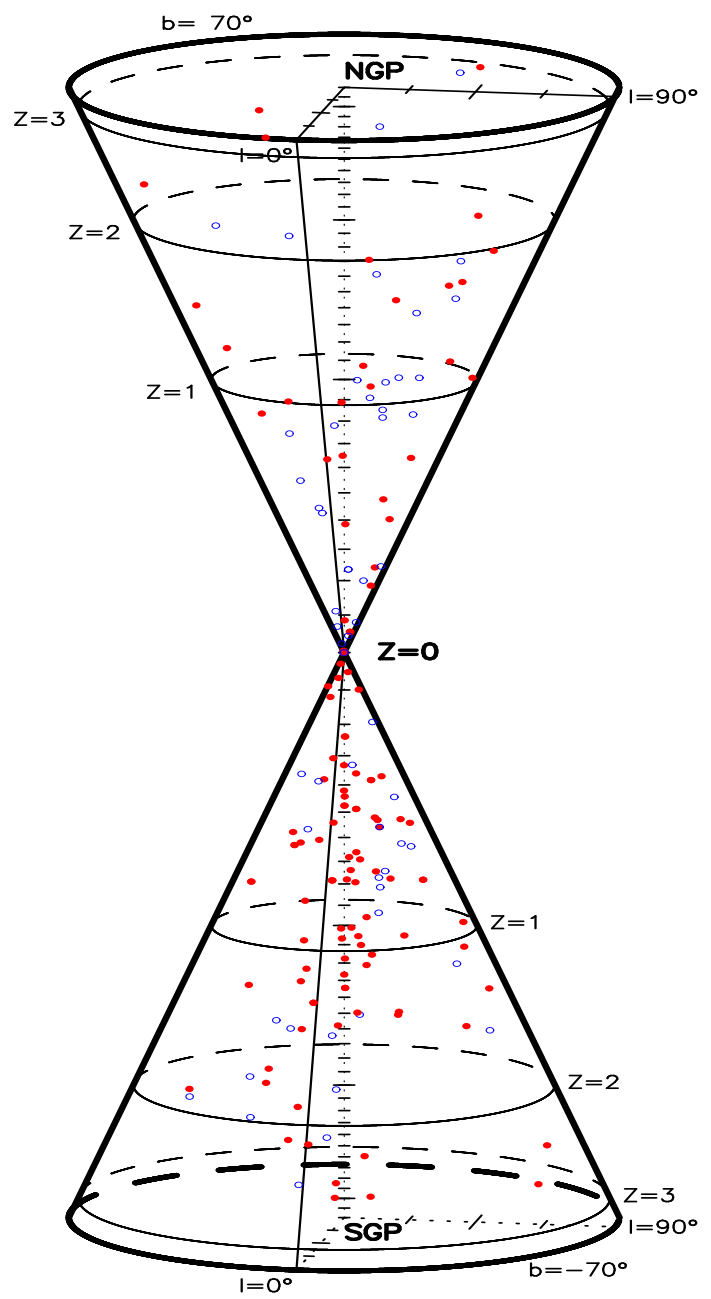

Figure 6: The space distribution of radio sources in the Galactic pole regions which we have their rotation measures observed. The red points stand for the positive RMs and blue ones for the negatives. It is clear that the rotation measures tend to be more positive (red) in the south Galactic pole and negative in the north pole, indicating the local vertical Galactic magnetic fields [8].

each redshift range, we require a large number of objects with measured RMs, so that effect of their intrinsic RMs does not dominant. Second, we do not have enough information about the electron density distribution, such as whether it is clouded in the intergalactic space and how it couples with magnetic fi elds $2 \$]$. To delineate the intergalactic magnetic fi elds, there is still a long way to go.

\section{Acknowledgments}

I am very grateful to colleagues who have collaborated with me: Dr. R.N. Manchester from Australia Telescope National Facility, CSIRO, Prof. G.J. Qiao from Peking University (China), Prof. Andrew Lyne from Jodrell Bank Observatory (UK), and Dr. Katia Ferriére from Observatory of Midi-Pyrénées (France), Dr. Willem van Straten from Swinborne University (AU). The author is supported by the National Natural Science Foundation of China (10521001 and 10773016). 


\section{References}

[1] Armstrong, J. W., Rickett, B. J., \& Spangler, S. R. 1995, ApJ, 443, 209

[2] Brown, J. C., Taylor, A. R., Wielebinski, R., \& Mueller, P. 2003, ApJ, 592, L29

[3] Brown, J. C., Haverkorn, M., Gaensler, B. M., \& et al. 2007, ApJ, 663, 258

[4] Freedman, W. L., Madore, B. F., Gibson, B. K., et al. 2001, ApJ, 553, 47

[5] Hamilton, P. A. \& Lyne, A. G. 1987, MNRAS, 224, 1073

[6] Han, J. L. 2004, In: The Magnetized Interstellar Medium, Copernicus GmbH, p.3

[7] Han, J. L., Ferriere, K., \& Manchester, R. N. 2004, ApJ, 610, 820

[8] Han, J. L., Man, H., Zhao J. 2008, in preparing

[9] Han, J. L., Manchester, R. N., Berkhuijsen, E. M., \& Beck, R. 1997, A\&A, 322, 98

[10] Han, J. L., Manchester, R. N., Lyne, A. G., \& Qiao, G. J. 2002, ApJ, 570, L17

[11] Han, J. L., Manchester, R. N., Lyne, A. G., Qiao, G. J., \& van Straten, W. 2006, ApJ, 642, 868.

[12] Han, J. L., Manchester, R. N., \& Qiao, G. J. 1999, MNRAS, 306, 371

[13] Han, J. L. \& Qiao, G. J. 1994, A\&A 288, 759

[14] Heiles, C. 1996b, in ASP Conf. Ser. 97: in: Polarimetry of the Interstellar Medium, 457

[15] Indrani, C. \& Deshpande, A. A. 1999, New Astronomy 4, 33

[16] Lyne, A. G. \& Smith, F. G. 1989, Nature, 218, 124

[17] Lyne, A. G. \& Smith, F. G. 1989, MNRAS, 237, 533

[18] Minter, A. H. \& Spangler, S. R. 1996, ApJ, 458, 194

[19] Ohno, H. \& Shibata, S. 1993, MNRAS, 262, 953

[20] Page, L. and Hinshaw, G. and Komatsu, E. et al. 2007, ApJS, 170, 335

[21] Rand, R. J. \& Kulkarni, S. R. 1989, ApJ, 343, 760

[22] Reich, W. 2007, "Galactic polarization surveys", in: Cosmic Polarization,ed. Roberto Fabbri, Publisher: Research Signpost

[23] Riess, A. G., Filippenko, A. V., Challis, P., et al. 1998, AJ, 116, 1009

[24] Strong, A. W., Moskalenko, I. V., \& Reimer, O. 2000, ApJ, 537, 763

[25] Sun, X. H., Reich, W., Waelkens, A., Enßlin, T. 2007, A\&A, in press

[26] Weisberg, J. M., Cordes, J. M., Kuan, et al. 2004, ApJS, 150, 317

[27] Wielebinski, R., Krause, F., 1993, A\&AR, 4, 449

[28] You, X.P., Han, J.L., Chen, Y. 2003, Acta Astronomica Sinica, Vol.44, Suppl. p.155 